\title{
Mental Lexicon and Words-Learning
}

\author{
Xiuying Zhang \\ Hubei Engineering University, Jiaotong Road 272, Xiaogan, Hubei ,432000
}

Keywords: Mental Lexicon, Words-Learning, Psycholinguistic Theory.

\begin{abstract}
How to enlarge our vocabulary when we learn a foreign language is an important topic. A lot of teachers and researchers and even learners have discussed about it. According to psycholinguistic theory, this article will talk about how we can remember words firmly in our mind after we master models of mental lexicon, which explains how words are stored and accessed in our mind.
\end{abstract}

\section{Introduction}

Words are to a foreign language what bricks are to a building. Therefore how to enrich vocabulary is a great concern for foreign language learning and teaching. Much research has been done in this field. However, most of the research has focused on improving the methods of teaching. (Huang 2003 Lu 2001, Wang 2001, Wang 2004) It's obvious that language especially words are mainly learned but not taught. Though some research has ever focused on words-learning, little has discussed about it from the view of mental lexicon. (Chen 2002 Tang 2002 Liu 2003) The models of organizations of mental lexicon and lexical access can provide us some implications on how to memorize words faster and well so that we will not forget them soon and even that we can know how to use them in proper places. The following paper will try to tell how we make use of these models of mental lexicon and models of lexical access in the process of words-learning.

\section{Mental lexicon and lexical access}

What is mental lexicon? Psycholinguists refer to the representation of words in permanent memory as mental lexicon. They study how words are stored in memory and how we retrieve information about words. When a given word in our lexicon has been found, the properties we associate with the word become available for use. These properties include the meaning of the word, its spelling and pronunciation, its relationship to others words, and related information. A word is a bundle of features. A word is part of a network. When we know a word, we know its phonological, morphological, syntactic, and semantic attributes. A word's meaning includes both sense and reference. Sense refers to a word's relationships with other words, whereas reference pertains to the relationships between a word and an object or event in the world.

The process by which we active our word knowledge is termed lexical access. Lexical access is influenced by the frequency of a word, its phonological and morphological attributes, whether it is ambiguous, and whether a semantically similar word has just been encountered. How is our mental lexicon organized in our brain? How can the theory of mental lexicon affect words-memorizing? Or how can we improve our vocabulary with the help of understanding how words are stored in memory and how we retrieve information about words.

\section{Organization of the mental lexicon and words-learning}

Several models of organization of mental lexicon have been formed in the process of psychological and linguistic research. And in fact each of them provides us an explanation on how words are stored in our brains and leaves us some implications for words-learning in foreign learning and teaching. 


\subsection{Semantic Feature Theory}

First, Semantic Feature Theory is assumed that by linguists. They claim that a category is defined by the sharing of features.

For example:

NOUNS:

man [MALE][ADULT]

husband [MALE][ADULT][MARRIED]

VERBS:

die [BECOME] [[NOT] [ALIVE]]

kill [CAUSE] [[BECOME] [[NOT] [ALIVE]]]

murder [CAUSE] [[BECOME] [[NOT] [ALIVE]]] AND

Learners of foreign language are told to remember words that are anonymous and antonymous to each other so that we can get a firm impression of the new words, and when we come across the old words we can think of the new words we have just learned This method of memorizing words comes from this semantic feature theory and it is not only used in foreign language learning but also used in native philology. For instance, when we were in primary school, we were taught to remember jijing and anjing together or renao and anjing together. However, not all psychologically real categories can be accurately defined with features! Such adjectives as ugly and beautiful, we can not use NOT UGLY or NOT BEAUTIFUL to indicate each other, therefore this theory can only be applicable to certain words-memorizing.

\subsection{Hierarchical Network Model}

Second, As Hierarchical Network Model describes, words are stored in the form of a semantic network, with some elements standing above or below other members of the network. Relations are represented by categories, such as relations of subordination and super-ordination, or by properties which indicate what characteristics may be attributed to the items at various levels in the network. The implications of this model for words-learning is similar to the Semantic Feature Theory since they both assume that words are stored in the form of a semantic network. The difference is that they use different semantic networks. So this model tells us that learners can remember the hyponymy and subordinate and super-ordinate words together. This method has similar limitations with the method deriving from Semantic Feature Theory. Not all the words are in a hierarchical network, so we need other models to supplement it.

\subsection{Prototype Theory}

Third, Prototype Theory claims that a category is defined by the sharing of "family resemblances" Rosch (1973), such as similarity to some other members in the category. There need not be a single feature or set of features shared by all the members. Prototype is the "best" member of the category, as determined by people's judgments; it is also typically named by the highest frequency word in its category. There are two important terms we should know in this Theory: taxonomy and basic level. A taxonomy is a system for classifying things. We'll introduce basic level in details since it is very important for us to learn words appropriately in foreign language learning.

Attributes are more likely to be stored at more familiar locations in the network. Most lexical hierarchies have a level, often near the middle, where most of the distinguishing features are assigned. This is called the basic-level terms. Basic level --- the level at which things are called, unless there are good reasons to do otherwise. For example, suppose a foreign learner of English points to Duke, and ask "What's that called in English?" You would probably respond, "It's a dog". You probably would not say, "It's an animal” or "It's a creature", even though these latter responses are equally "true". And dog is the word that you would almost certainly teach to a young child.

What is it that confers salience on one particular level in a taxonomy? The question was addressed by Rosch (1975) and by Rosch and Mervis (1975). Rosch presented subjects with category names, at different levels in a taxonomy, and ask them to list all the characteristic features of the categories. It turned out that units at the basic level were characterised by 
(1) a relatively large number of features.

(2) basic level terms tended not to share features amongst themselves.

(3) Units below the basic level shared a significant number of attributes amongst themselves,

While units above the basic level exhibited very few nameable attributes at all. Rosch therefore identified the basic level in terms of its informativity. To call something by a name below the basic level adds relatively little information. Units above the basic level are generally so schematic, that they are applicable to a very wide range of instances. To call something by a name above the basic level actually tells you very little about the entity. Basic level terms - such as dog, chair, hammer tend to be short, morphologically simple, and of high frequency of occurrence. Terms below the basic level are often morphologically complex (deck-chair, claw hammer), while terms above the basic level often have a scientific, or "bookish" feel to them (mammal, implement.). (Liang \& Feng 2003). The prototype model tells that when we learn words we may follow a proper sequence. Generally speaking, we should first learn those basic words in each taxonomy just as children acquire their mother tongue. Words of basic level tend to be short, morphologically simple and of high frequency of occurrence, so they are both simpler for us to remember and most applicable in our reading, writing, speaking and listening. Those learners who want to jump over the basic levels words and try to memorize some dictionaries so as to enrich vocabulary in a short time should give up their idea, on the contrary they should follow teaching syllable at first so that they can master the basic level words well, which will benefit them in an unconscious way. They should bear in mind: Haste makes waste.

\subsection{Spreading activation model}

Words are represented in the mental lexicon in a network, but the organization is not strictly hierarchical. In contrast, the organization is closer to a web of interconnecting nodes. Activation begins at a single node and then spreads in parallel throughout the network. (Collins and Loftus, 1975) Advantage of this model is that it is a great step forward from the rigid hierarchical model and prototype theory. It shows that each word may be interrelated to several semantic networks. But there are still are limitations: it is a model of concept rather than words because very little attention is paid to phonological, syntactic and morphological aspects of words.

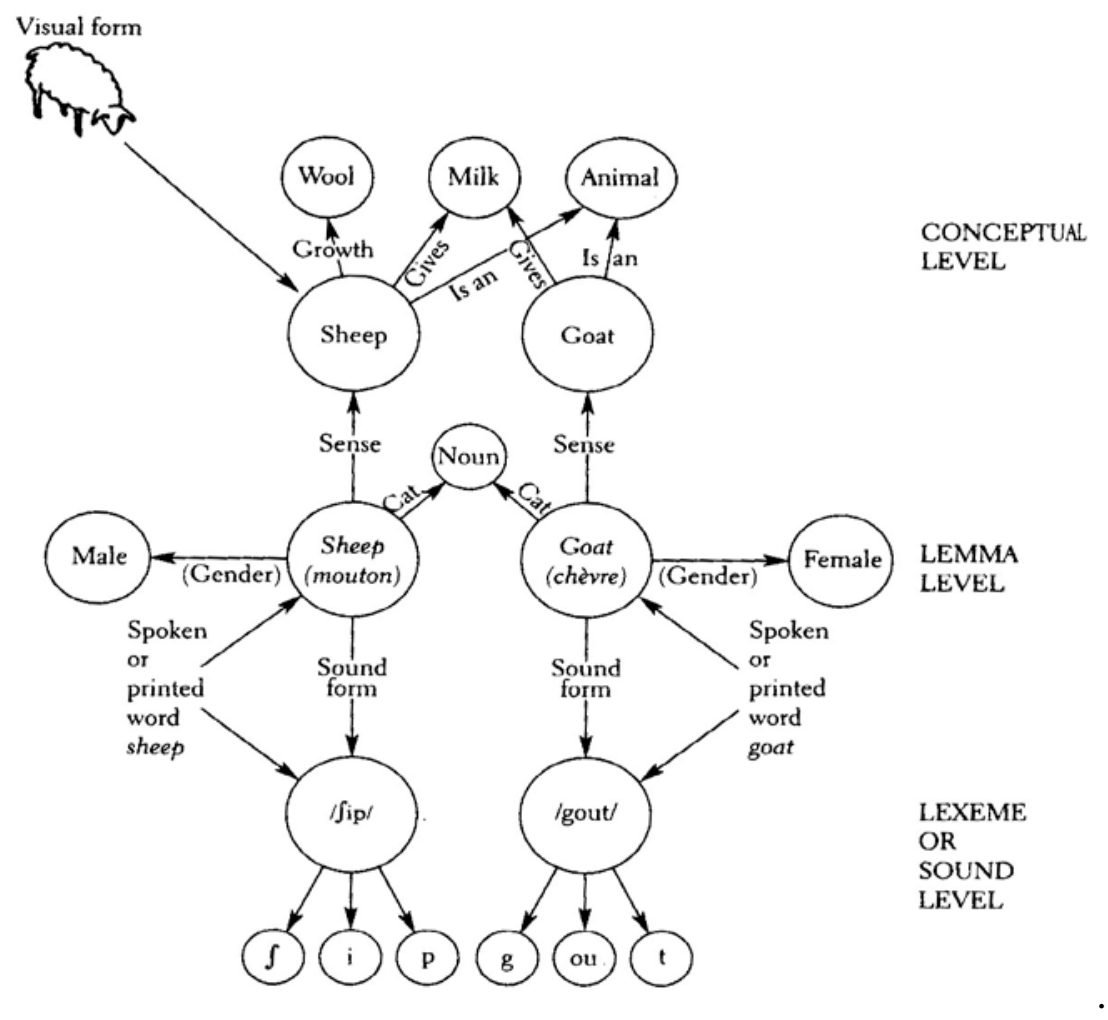

Figure 3 A part of lexical network 
This model is improved by Bock and Levelt (1994). They incorporates lexical as well as conceptual aspects. Our knowledge of words exists at three different levels: the conceptual level, the syntactic level, the lexeme level. The conceptual level consists of nodes representing concept. The second level refers to syntactic aspects of word knowledge. The lexeme level captures a word's phonological properties or how a word sounds Spreading activation models of the lexicon that incorporate conceptual, syntactic and phonological knowledge appear to offer the most realistic picture currently available of the mental lexicon. Consequently it provides us very useful implications: since our knowledge of words exists at three levels: syntactic, lexical, conceptual, each of them gives us some complication. Syntactic level tells us we should try to learn each word especially verb in a short context so that we can know how to use them in a proper place. Lexical level tells us it is the best way to use more of our senses as possible as we can when we memorize words. Conceptual level inspires us to memorize words while thinking of the event or image related.

\section{Models of lexical access}

Lexical access is the way we active or retrieve our words when we come across them in reading or listening or when we want to use them in speaking or writing. There are several models of lexical access. It is also very helpful if we can apply the knowledge of these models of lexical access into words-learning.

\subsection{Search Model}

Search model is brought by Forster. (1976). And it is a type of two -stage processing model, where the first stage is completed before the second stage begins: it is serial rather than parallel in its mode of operation. The two stages are-search, followed by matching

The first stage - the search is carried on: this search is ordered by frequency of the lexical items in the languages, beginning with the most frequent items, then successively down through the listing until the lowest-frequency items are reached.

The second stage- according to the analyzed properties of the input word available, we search through the frequency listing for a match with those properties. While we don't access to the full lexical item itself, but an abstract location marker, which tells us where the full lexical item is stored. We then use it to gain our access to the lexical item.

\section{Access files- (catalogues)}

(a) Orthographic access file

(b) Phonological access file

(c) Semantic/ syntactic file

The access files are linked to a unitary master file, whose function is to represent all aspects of all the entries in the lexicon, their phonological, orthographic and syntactic-semantic properties. Once an entry in the master file is accessed, it is available for implementing any sort of response-speaking the word, writing the word, and understanding the word. This unitary master file again proves that we should try to use all our senses to remember words: when we read aloud with our eyes working, we'd better write with our hand and think with our brain. Since experiment proves that high-frequency words will be accessed faster than low-frequency words, learners should try to practice more so that we can access words faster. It also testifies to the proverb: Practice makes perfect.

\subsection{Logogen Model}

Logogen model is first brought out by Morton. (1969). Each word or morpheme in the lexicon is represented as a logogen, which specifies the word's various attributes: semantic, orthographic, phonological, and so on. The logogen is activated in either two ways:

By sensory input--As orthographic or phonological features of the input stimulus are detected, they are matched to the logogen. The logogen functions as a scoreboard or counter; when the counter rises above a predesignated threshold, the item is recognized. 
By contextual information - the semantic and syntactic structure of a sentence may influence the activation of the logogen for a given word.

E.g, her closest relative was appointed as her legal guardian.

The activation of the earlier words influences the logogen for the final word, temporarily lowering its threshold. Thus it's easier to recognize guardian in the context than if presented isolation. The two ways are assumed to work in parallel; sensory and contextual matches increase the same counter. Thus, when many sensory word will be activated even if it is somewhat unexpected. Similarly, an expected word will be activated even if presented in dim light. Of course, if both sensory and contextual features are detected, then the word is easily detected.

Search model divided lexical access into two stages, while Logogen model divides lexical access into two levels. They are different, however, they provide us the same implication as Search model does: sensory input reminds us the importance of using senses simultaneously; contextual information encourages us to remember words in context not separately.

\subsection{Cohort Model-}

Cohort Model is founded by Marslen-Wilson\&Welsh in 1978. It's designed specifically to account for auditory word recognition. The spoken word recognition occurs in three stages: First, on the basis of an acoustic-phonetic analysis of the input, a set of lexical candidates is activated. -word initial cohort. Second, one member of the cohort is selected for further analysis. Finally, the selected lexical item is integrated into the ongoing semantic and syntactic context.

E.g. Angela misplaced her ba...

As for the first stage, the initial activation of items is done in a strictly bottom-up fashion. Upon hearing the above sentence, bag, bat, bath, bass, and others would be available for selection. This cohort is then submitted to the selection process. The activation levels of different items in the cohort vary as a function of their similarity to the incoming signal. Items that are very similar to the signal remain strongly activated, while the level of others drops off. Thus, some of the initial candidates in the cohort are progressively eliminated. The elimination takes place in one of two ways: either the context of a spoken sentence narrows the initial cohort or candidates are discarded as more phonological information comes in. eventually, only one candidate will remain.

Since Cohort Model designed specifically to account for auditory word recognition, it

may be better positions to explain the full range of factors that influence lexical access.

The process of accessing or retrieving lexical information from memory is influenced by a number of factors. Among such factors are the frequencies of the words, its syntactic category, morphological complexity, whether a semantically related word has just been encountered, and whether the word is ambiguous.

\section{Conclusion}

We all know that no word can be remembered firmly if we separate it from other words completely. Words of all kinds and levels are interconnected. Therefore, we must associate each word with other words or place it in a short context or even a discourse when we try to remember it. From the above discussions, we can know that each model provides us some implications for words-learning, however, no model can provide us a perfect and omnipotent method of memorizing words, but we can find each implication is supplement to others. As far as the writer is concerned, the improved Spreading Activation Model of mental lexicon leaves us the most important clues that we should learn words by trying using all our senses together and memorizing each word in a context as possible as we can.

\section{References}

[1] Ahrens, Kathleen. "Lexical ambiguity resolution: languages, tasks, and timing." Syntax and Semantics.1998,33 (1).

[2] Forster, K. I. "Accessing the mental lexicon." In R. J. Wales and E. Walker [eds] New 
Approaches to Language Mechanisms, 1976.

[3] Liu l. Semantic theory and words-learning. Journal of Tangshan College.2003,17(3)

[4] MacKay, D. G. "Derivational rules and the internal lexicon." Journal of Verbal Learning and Verbal Behavior 1978,17 (2).

[5] Wang C. Mental lexicon and words teaching. Social Science Review 2004,19(4).

[6] Zhou Xiaolin and Marslen-Wilson, William "Words, morphemes and syllables in the Chinese mental lexicon." Language and Cognitive Processes 1994, 9(3). 